\title{
SYNTHESIS AND ANTIFUNGAL ACTIVITY OF NEW O-ALKYLAMIDOXIMES
}

\author{
Maria Verônica Sales BARBOSA ${ }^{1}$ (i), Alana Karoline Penha NASCIMENTO² (D), \\ Rodrigo Ribeiro Alves CAIANA ${ }^{2}$ (D) , Cosme Silva SANTOS ${ }^{1}$ (D) , Wylly Araújo de OLIVEIRA ${ }^{3}$ (D), \\ Paulo Henrique MENEZES ${ }^{4}$ (D), Juliano Carlo Rufino FREITAS ${ }^{3}$ (iD)
}

\footnotetext{
${ }^{1}$ Postgraduate Program in Chemistry, Rural Federal University of Pernambuco, Recife, Pernambuco, Brazil.

2 Postgraduate Program in Natural Sciences and Biotechnology, Rural Federal University of Campina Grande, Cuité, Paraíba, Brazil.

${ }^{3}$ Education and Health Center, Federal University of Campina Grande, Cuité, Paraíba, Brazil.

${ }^{4}$ Department of Chemistry, Federal University of Pernambuco, Recife, Pernambuco, Brazil.
}

Corresponding author:

Juliano Carlo Rufino Freitas

Email: julianocrufino@pq.cnpq.br

How to cite: BARBOSA, M.V.S., et al. Synthesis and antifungal activity of new O-alkylamidoximes. Bioscience Journal. 2021,37, e37049. https://doi.org/10.14393/BJ-v37n0a2021-54171

\begin{abstract}
The continuous prospection for molecules that may be useful in the development of new therapeutic agents is a highly relevant issue, mainly because the launch of new drugs on the market does not accompany the emergence of new resistant microorganisms. In this context, this work describes the synthesis of new $\mathrm{O}$ alkylamidoximes and the evaluation of its antifungal activity. The new $\mathrm{O}$-alkylamidoximes were prepared using easy synthetic protocols and tested against three Candida species using the broth microdilution method. The synthesized compounds were obtained in moderate to good yields in high purity and without any observable decomposition. All tested compounds shown moderate antifungal activity against at least one strain of Candida. Despite the moderate activity of the new compounds, this was the first report involving the antifungal activity of O-alkylamidoximes. In view of the low chemotherapy arsenal and the development of fungal strains resistant to traditional antifungal agents, the present study opens new possibilities for the preparation of a new class of more active antifungal agents.
\end{abstract}

Keywords: Antifungal. Candida. $\mathrm{O}$-alkylation. $\mathrm{O}$-alkylamidoximes.

\section{Introduction}

Fungi are widespread in the environment and are found in a variety of habitats (Prasad and Kapoor 2004). These microorganisms make up the normal human microbiota and are commonly found in the skin, gastrointestinal, respiratory, and genitourinary tracts (Kapitan et al. 2019). However, in immunodeficient individuals such as transplanted (Eades and Armstrong-James 2019) and HIV-infected (Wang et al. 2017), the contact with pathogenic fungi (Capote et al. 2016) can lead to severe infections. Thus, fungal infections have been a major concern for health regulators, special due to the considerable morbidity and mortality rates, contributing factors to the increased health costs (Silva et al. 2012).

Among the different etiological agents involved in this type of pathology, Candida spp. are the most prevalent, representing approximately $19 \%$ of all infections worldwide in intensive care units (Irfan et al. 2017). It is also estimated that around 25 to $75 \%$ of healthy individuals to have Candida spp colonies in their normal microbiota (Costello et al. 2009). However, different species of this genus are often identified as the cause of invasive infections in humans, with special attention to $C$. albicans due to its prevalence in both 
healthy patients and morbid individuals. In addition, $C$. albicans is present in approximately $70 \%$ of clinical isolates, being the number of cases generated by other Candida species (examples: C. glabrata, C. tropicalis, and C. parapsilosis) continuously growing (Kung et al. 2016).

Associated with these problems are the events of resistance of various microorganisms to antifungal agents (Berman and Krysan 2020). Currently, there is a major concern about the growing cases of microbial resistance worldwide, since, in the absence of efficient antimicrobial agents, it is estimated that incurable diseases will be responsible for about 10 million casualties by 2050 (De Kraker et al. 2016). Thus, the continuous prospection for molecules that may be useful in the development of new therapeutic agents is a highly relevant issue and has been encouraged, mainly due to the appearance of new drugs on the market not to follow the appearance of new resistant microorganisms (Pfaller 2012; Lee and Lee 2018).

In addition to the events of antimicrobial resistance, the limited therapeutic arsenal, the concern about toxicity, drug interactions, and low bioavailability presented by current antifungal agents are issues that deserve attention (Cavaleiro et al. 2006; Spitzer et al. 2017; Wiederhold 2017; Costa-de-Oliveira and Rodrigues 2020). The scenario stimulates the development of new molecules to address these problems. However, the development of new antifungals is challenging, since fungi are eukaryotic organisms which, when compared to human host cells, present only a few different targets (Mccarthy et al. 2017). In this work, $O$-alkylamidoximes, structurally simpler molecules when compared to other antifungal agents have virtually untapped biological potential. Reports of the biological activities for this class of molecules are rare, however, they can act as antipneumocystic (Boykin et al. 1996), antiplatelet (Rehse and Brehme 1998), antithrombotic (Rehse and Brehme 1998), antibacterial (Zhan-Tao et al. 2005), and as poly (ADP-ribose) polymerase-1 (PARP-1) enzyme inhibitors (Szabados et al. 2000). In this context, in this work is described the synthesis of new $O$-alkylamidoximes, and the evaluation of its antifungal activity against three different Candida species.

\section{Material and Methods}

\section{Reagent materials and equipment}

The solvents were distilled before use as reported in the literature (Armarego 2017). Ethanol was dried by distillation from metallic magnesium. Ethyl acetate and hexane were distilled using a vigreux column. All commercially available reagents were used as received. The reactions were monitored by thinlayer chromatography (TLC) using different eluent systems. Compounds were visualized with UV light. Column chromatographic purification was performed using silica gel 60 (70-230 mesh) unless indicated otherwise (Still et al. 1978). All compounds purified by crystallization or chromatography were pure enough for use in other experiments.

The IR spectra were recorded on a Fourier Spectrum 400 FT-IR/FT-NIR Spectrometer Model Perkin Elmer, the samples being prepared as $\mathrm{KBr}$ pellet or thin films. The carbon, hydrogen, and nitrogen contents of the compounds were determined by the Dynamic Flash Combustion technique, in a CHNS-O elementary analyzer, CE Instruments, model EA $1110 .{ }^{1} \mathrm{H}$ NMR data were recorded at $400 \mathrm{MHz}$ using a Varian UNITY PLUS spectrometer. ${ }^{1} \mathrm{H}$ and ${ }^{13} \mathrm{C}$ NMR spectra were obtained on a Varian Unity Plus-400 spectrometer using $\mathrm{CDCl}_{3}$ or DMSO- $d_{6}$ as the solvents, and calibrated for the solvent signal and tetramethylsilane (TMS) as internal reference. Coupling constants $(J)$ were reported in Hertz $(\mathrm{Hz})$.

\section{General procedure for the synthesis of Amidoximes 2a-h}

The synthesis of amidoximes 2a-h was based on the methodology previously described in the literature (Barros et al. 2011). To a flask containing hydroxylamine hydrochloride $(2.08 \mathrm{~g}, 30 \mathrm{mmol})$ and sodium carbonate $(1.6 \mathrm{~g}, 15 \mathrm{mmol})$ at $25^{\circ} \mathrm{C}$ was added distilled water $(30 \mathrm{~mL})$. The mixture was stirred and then a solution of the appropriate nitrile $1 \mathrm{a}-\mathrm{h}(10 \mathrm{mmol})$ in ethanol $(40 \mathrm{~mL})$ was added dropwise. The reaction mixture was placed in an ultrasonic bath at $55 \pm 5{ }^{\circ} \mathrm{C}$ and monitored by TLC. After completion, the solvents were removed in vacuo. The residual solid was extracted with ethyl acetate $(3 \times 25 \mathrm{~mL})$ and the combined organic phases dried over anhydrous $\mathrm{Na}_{2} \mathrm{SO}_{4}$. Filtration, following removal of the solvent under 
reduced pressure, provided the corresponding amidoximes 1a-h which were purified by crystallization using hexane/chloroform (10:90) system.

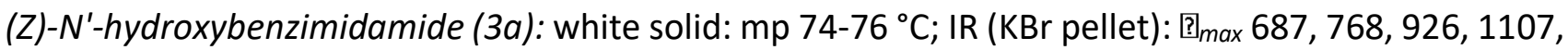
$1384,1446,1499,1590,1645,2361,2893,3059,3212,3356,3448, \mathrm{~cm}^{-1} ;{ }^{1} \mathrm{H}$ NMR $\left(400 \mathrm{MHz}, \mathrm{DMSO}-d_{6}\right) \delta$ $5,81\left(2 \mathrm{H}, \mathrm{s}, \mathrm{NH}_{2}\right), 7.38-7.36\left(3 \mathrm{H}, \mathrm{m}, \mathrm{H}_{\text {Aryl }}\right), 7.69-7.67\left(2 \mathrm{H}, \mathrm{m}, \mathrm{H}_{\text {Aryl }}\right), 9.63(1 \mathrm{H}, \mathrm{s}, \mathrm{OH}) ;{ }^{13} \mathrm{C} \mathrm{NMR}(100 \mathrm{MHz}, \mathrm{DMSO}-$ $\left.d_{6}\right) \delta 125.4,128.1,128.9,133.4,150.8$. Compound data are in accordance with the literature (Ozcan et al. 2013; Andrade et al. 2016).

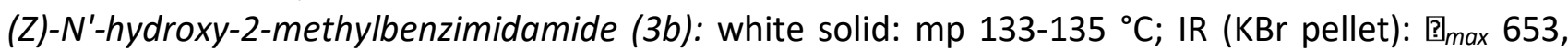
$722,770,903,1107,1374,1437,1580,1651,2921,3152,3197,3363,3479 \mathrm{~cm}^{-1}$; ${ }^{1} \mathrm{H}$ NMR (400 MHz, DMSO$\left.d_{6}\right) \delta 2.34\left(3 \mathrm{H}, \mathrm{s}, \mathrm{CH}_{3 \text { aryl }}\right), 5.70\left(2 \mathrm{H}, \mathrm{s}, \mathrm{NH}_{2}\right), 7.22-7.16\left(2 \mathrm{H}, \mathrm{m}, \mathrm{H}_{\text {Aryl }}\right), 7.28-7.25\left(2 \mathrm{H}, \mathrm{m}, \mathrm{H}_{\text {Aryl }}\right), 9.30(1 \mathrm{H}, \mathrm{s}, \mathrm{OH})$; ${ }^{13} \mathrm{C}$ NMR $\left(100 \mathrm{MHz}, \mathrm{DMSO}-d_{6}\right) \delta 19.7,125.3,128.4,128.8,130.1,134.3,136.2,152.3$. Compound data are in accordance with the literature (Andrade et al. 2016; Tarasenko et al. 2017).

(Z)-N'-hydroxy-3-methylbenzimidamide (3c): white solid: $\mathrm{mp}$ 133-135 ㅇ; IR (KBr pellet): 目ax 700, $793,892,931,1085,1389,1586,1647,2360,2921,3039,3200,3357,3454 \mathrm{~cm}^{-1}$; ${ }^{1} \mathrm{H}$ NMR(400 MHz, DMSO$\left.d_{6}\right) \delta 2.32\left(3 \mathrm{H}, \mathrm{s}, \mathrm{CH}_{3 \text { aryl }}\right), 5.75\left(2 \mathrm{H}, \mathrm{s}, \mathrm{NH}_{2}\right), 7.21-7.17\left(2 \mathrm{H}, \mathrm{m}, \mathrm{H}_{\text {Aryl }}\right), 7.28-7.27\left(2 \mathrm{H}, \mathrm{m}, \mathrm{H}_{\text {Aryl }}\right), 9.57(1 \mathrm{H}, \mathrm{s}, \mathrm{OH})$; ${ }^{13} \mathrm{C}$ NMR $\left(100 \mathrm{MHz}, \mathrm{DMSO}-d_{6}\right) \delta 21.1,122,6,125.9,129.7,129.5,133.3,137.1,150.9$. Compound data are in accordance with the literature (Andrade et al. 2016).

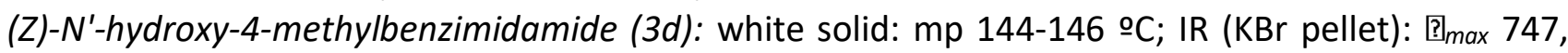
823, 936, 1099, 1391, 1418, 1588, 1664, 2916, 3049, 3367, $3500 \mathrm{~cm}^{-1} ;{ }^{1} \mathrm{H}$ NMR (400 MHz, DMSO-d $) \delta 2.31$ $\left(3 \mathrm{H}, \mathrm{s}, \mathrm{CH}_{3 \text { aryl }}\right), 5.73\left(2 \mathrm{H}, \mathrm{s}, \mathrm{NH}_{2}\right), 7.17\left(2 \mathrm{H}, \mathrm{d}, J=8.2 \mathrm{~Hz}, \mathrm{H}_{\text {Aryl }}\right), 7.56\left(2 \mathrm{H}, \mathrm{d}, J=8.2 \mathrm{~Hz}, \mathrm{H}_{\text {Aryl }}\right), 9.52(1 \mathrm{H}, \mathrm{s}, \mathrm{OH})$; ${ }^{13} \mathrm{C}$ NMR $\left(100 \mathrm{MHz}\right.$, DMSO- $\left.d_{6}\right) \delta 20.8,125.3,128.6,130.5,138.2,150.8$, Compound data are in accordance with the literature (Andrade et al. 2016; Tarasenko et al. 2017).

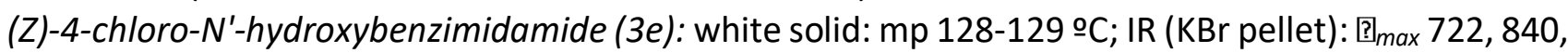
$920,1087,1380,1497,1589,1655,1918,2361,2893,3053,3152,3346,3468 \mathrm{~cm}^{-1} ;{ }^{1} \mathrm{H}$ NMR $(400 \mathrm{MHz}$, DMSO- $\left.d_{6}\right) \delta 5.86\left(2 \mathrm{H}, \mathrm{s}, \mathrm{NH}_{2}\right), 7.43\left(2 \mathrm{H}, \mathrm{d}, J=8.6 \mathrm{~Hz}, \mathrm{H}_{\text {Aryl }}\right), 7.69\left(2 \mathrm{H}, \mathrm{d}, J=8.6 \mathrm{~Hz}, \mathrm{H}_{\text {Aryl }}\right), 9.73(1 \mathrm{H}, \mathrm{s}, \mathrm{OH}) ;{ }^{13} \mathrm{C}$ NMR $\left(100 \mathrm{MHz}, \mathrm{DMSO}-d_{6}\right) \delta 127.1,128.1,133.2,133.4,149.9$. Compound data are in accordance with the literature (Ozcan et al. 2013; Andrade et al. 2016; Tarasenko et al. 2017).

(Z)-4-bromo-N'-hydroxybenzimidamide (3f): white solid: $\mathrm{mp} 140-141$ ㅇ; $\mathrm{IR}$ (KBr pellet): ? $]_{\max } 720,833$, $921,1010,1069,1380,1492,1583,1657,1914,2890,3050,3108,3356,3472 \mathrm{~cm}^{-1} ;{ }^{1} \mathrm{H} \mathrm{NMR}(400 \mathrm{MHz}$, DMSO- $\left.d_{6}\right) \delta 5,86\left(2 \mathrm{H}, \mathrm{s}, \mathrm{NH}_{2}\right), 7,57\left(2 \mathrm{H}, \mathrm{d}, J=8,6 \mathrm{~Hz}, \mathrm{H}_{\text {Aryl }}\right), 7,63\left(2 \mathrm{H}, \mathrm{d}, J=8,6 \mathrm{~Hz}, \mathrm{H}_{\text {Aryl }}\right), 9,74(1 \mathrm{H}, \mathrm{s}, \mathrm{OH}) ;{ }^{13} \mathrm{C}$ NMR $\left(100 \mathrm{MHz}\right.$, DMSO- $d_{6} \delta 122.1,127.4,131.0,132.5,149.9$. Compound data are in accordance with the literature (Li et al. 2013).

(Z)-N'-hydroxy-4-nitrobenzimidamide (3g): white solid: mp 180-181 ㄷ; IR (KBr pellet): 目 ${ }_{\max }$ 700, 810, $860,922,1105,1337,1512,1596,1657,2844,3114,3180,3351 \mathrm{~cm}^{-1} ;{ }^{1} \mathrm{H}$ MNR (400 MHz, DMSO-d $d_{6} \delta 6,08$ $\left(2 \mathrm{H}, \mathrm{s}, \mathrm{NH}_{2}\right), 7,97\left(2 \mathrm{H}, \mathrm{d}, J=9,0 \mathrm{~Hz}, \mathrm{H}_{\text {Aryl }}\right), 8,25\left(2 \mathrm{H}, \mathrm{d}, J=9,0 \mathrm{~Hz}, \mathrm{H}_{\text {Aryl }}\right), 10,16(1 \mathrm{H}, \mathrm{s}, \mathrm{OH}) ;{ }^{13} \mathrm{C} \mathrm{NMR}(100 \mathrm{MHz}$, DMSO- $\left.d_{6}\right) \delta 123.4,126.4,139.5,147.5,149.4$. Compound data are in accordance with the literature (Li et al. 2013; Ozcan et al. 2013).

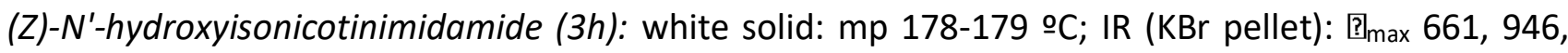
$1085,1218,1383,1413,1539,1591,1630,2750,2795,3051,3156,3308,3460 \mathrm{~cm}^{-1} ;{ }^{1} \mathrm{H} \mathrm{NMR}(400 \mathrm{MHz}$,

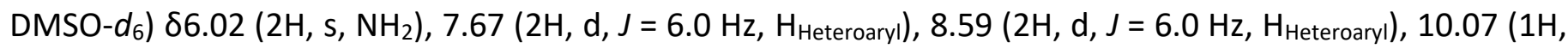
$\mathrm{s}, \mathrm{OH}) ;{ }^{13} \mathrm{C}$ NMR $\left(100 \mathrm{MHz}, \mathrm{DMSO}-d_{6}\right) \delta 119.6,140.5,148.9,149.7$. Compound data are in accordance with the literature (Ozcan et al. 2013).

\section{General procedure for preparing 0-alkylamidoximes (3a-h)}

To the flask was added the appropriate amidoxime $2 \mathrm{a}-\mathrm{h}(0.5 \mathrm{mmol})$, sodium hydroxide $(32 \mathrm{mg}, 0.8$ $\mathrm{mmol})$, and DMSO ( $4 \mathrm{~mL})$. The mixture was stirred for 5 minutes and then bromoacetaldehyde diethyl acetal (118.2 $\mathrm{mg}, 0.6 \mathrm{mmol}$ ) was added. The reaction mixture was stirred at room temperature and monitored by TLC [hexane/ethyl acetate (40:60)]. After completion, the reaction mixture was extracted with ethyl acetate $\left(3 \times 20 \mathrm{~mL}\right.$ ). The organic phase was separated, dried over anhydrous $\mathrm{Na}_{2} \mathrm{SO}_{4}$, filtered and the solvent removed under reduced pressure. The crude product was purified by a flash column chromatography [hexane/ ethyl acetate (95:5)] to yield compounds 3a-h. 
(Z)-N'-(2,2-diethoxyethoxy)benzimidamide (3a): Colorless oil; IR (KBr pellet): [ $\max _{\max } 695,772,897,1070$, $1265,1395,1444,1502,1634,1959,2882,2930,2974,3058,3370,3488 \mathrm{~cm}^{-1}$; ${ }^{1} \mathrm{H}$ NMR $\left(400 \mathrm{MHz}, \mathrm{CDCl}_{3}\right) \delta$ $1.24\left(6 \mathrm{H}, \mathrm{t}, J=7.2 \mathrm{~Hz},-\mathrm{CH}_{2} \mathrm{CH}_{3}\right), 3.60\left(2 \mathrm{H}, \mathrm{dq}, J=9.2\right.$ and $\left.7.2 \mathrm{~Hz},-\mathrm{CH}_{2} \mathrm{CH}_{3}\right), 3.76(2 \mathrm{H}, \mathrm{dq}, J=9.2$ and $7.2 \mathrm{~Hz}$, $\left.\mathrm{CH}_{2} \mathrm{CH}_{3}\right), 4.12\left(2 \mathrm{H}, \mathrm{d}, J=5.6 \mathrm{~Hz},-\mathrm{CH}_{2} \mathrm{CH}-\right), 4.87\left(1 \mathrm{H}, \mathrm{t}, J=5.6 \mathrm{~Hz},-\mathrm{CH}_{2} \mathrm{CH}-\right), 4.87\left(2 \mathrm{H}, \mathrm{br}, \mathrm{NH}_{2}\right), 7.44-7.37(3 \mathrm{H}$, $\left.\mathrm{m}, \mathrm{H}_{\text {Aryl }}\right), 7.64-7.62\left(2 \mathrm{H}, \mathrm{m}, \mathrm{H}_{\text {Aryl }}\right) ;{ }^{13} \mathrm{C}$ NMR $\left(100 \mathrm{MHz} \mathrm{CDCl}_{3}\right), \delta 15.4,62.5,73.5,100.4,125.9,128.6,129.9$, 132.4,152.3. Compound data are in accordance with the literature (Veerman et al. 2016).

(Z)-N'-(2,2-diethoxyethoxy)-2-methylbenzimidamide (3b): Colorless oil; IR (KBr pellet): ? $?_{\max } 726,762$, $889,1066,1387,1446,1632,2880,2930,2973,3354,3482 \mathrm{~cm}^{-1} ;{ }^{1} \mathrm{H}$ NMR $\left(400 \mathrm{MHz}, \mathrm{CDCl}_{3}\right) \delta 1.26(6 \mathrm{H}, \mathrm{t}, J=$ $\left.6.8 \mathrm{~Hz},-\mathrm{CH}_{2} \mathrm{CH}_{3}\right), 2.44\left(3 \mathrm{H}, \mathrm{s}, \operatorname{Aryl}-\mathrm{CH}_{3}\right), 3.61\left(2 \mathrm{H}, \mathrm{dq}, J=9.2\right.$ and $\left.6.8 \mathrm{~Hz},-\mathrm{CH}_{2} \mathrm{CH}_{3}\right), 3.76(2 \mathrm{H}, \mathrm{dq}, J=9.2$ and $\left.6.8 \mathrm{~Hz}, \mathrm{CH}_{2} \mathrm{CH}_{3}\right), 4.07\left(2 \mathrm{H}, \mathrm{d}, J=5.2 \mathrm{~Hz},-\mathrm{CH}_{2} \mathrm{CH}-\right), 4.83\left(2 \mathrm{H}, \mathrm{br}, \mathrm{NH}_{2}\right), 4.85\left(1 \mathrm{H}, \mathrm{t}, J=5.2 \mathrm{~Hz},-\mathrm{CH}_{2} \mathrm{CH}\right), 7.23-$ $7.18\left(2 \mathrm{H}, \mathrm{m}, \mathrm{H}_{\text {Aryl }}\right), 7.31-7.27\left(1 \mathrm{H}, \mathrm{m}, \mathrm{H}_{\text {Aryl }}\right), 7.38-7.35\left(1 \mathrm{H}, \mathrm{m}, \mathrm{H}_{\text {Aryl }}\right) ;{ }^{13} \mathrm{C}$ NMR $\left(100 \mathrm{MHz} \mathrm{CDCl}_{3}\right) \delta 15.4,19.6$, $62.6,73.3,100.5,125.8,128.8,129.5,130.6,132.5,136.7,153.0$. Anal. calcd for $\mathrm{C}_{14} \mathrm{H}_{22} \mathrm{~N}_{2} \mathrm{O}_{3}: \mathrm{C}, 63.13 \% ; \mathrm{H}$, 8.33\%; N, 10.52\%. Obtained: C, 62.89\%; H, 8.21\%; N, 10.36\%.

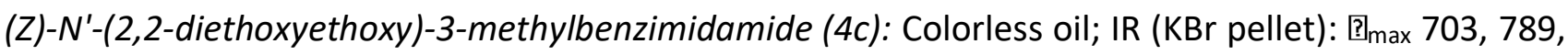
$896,1070,1389,1588,1633,2361,2878,2929,2974,3366,3486 \mathrm{~cm}^{-1} ;{ }^{1} \mathrm{H}$ NMR $\left(400 \mathrm{MHz}, \mathrm{CDCl}_{3}\right) \delta 1.24(6 \mathrm{H}$, $\left.\mathrm{t}, J=7.2 \mathrm{~Hz},-\mathrm{CH}_{2} \mathrm{CH}_{3}\right), 2.37\left(3 \mathrm{H}, \mathrm{s}, \mathrm{CH}_{3}\right), 3.61\left(2 \mathrm{H}, \mathrm{dq}, J=9.2\right.$ and $\left.7.2 \mathrm{~Hz},-\mathrm{CH}_{2} \mathrm{CH}_{3}\right), 3.74(2 \mathrm{H}, \mathrm{dq}, J=9.2$ and $\left.7.2 \mathrm{~Hz},-\mathrm{CH}_{2} \mathrm{CH}_{3}\right), 4.11\left(2 \mathrm{H}, \mathrm{d}, J=5.6 \mathrm{~Hz},-\mathrm{CH}_{2} \mathrm{CH}-\right), 4.83\left(2 \mathrm{H}, \mathrm{br} \mathrm{s}, \mathrm{NH}_{2}\right), 4.86\left(1 \mathrm{H}, \mathrm{t}, J=5.6 \mathrm{~Hz},-\mathrm{CH}_{2} \mathrm{CH}\right), 7.29-$ $7.21\left(2 \mathrm{H}, \mathrm{m}, \mathrm{H}_{\text {Aryl }}\right), 7.46-7.39\left(2 \mathrm{H}, \mathrm{m}, \mathrm{H}_{\text {Aryl }}\right) ;{ }^{13} \mathrm{C} \mathrm{NMR}\left(100 \mathrm{MHz}, \mathrm{CDCl}_{3}\right) \delta 15.4,21.3,62.4,73.4,100.4,122.9$, $126.5,128.5,130.7,132.3,138.3,152.6$. Anal. calcd for $\mathrm{C}_{14} \mathrm{H}_{22} \mathrm{~N}_{2} \mathrm{O}_{3}$ : $\mathrm{C}, 63.13 \% ; \mathrm{H}, 8.33 \% ; \mathrm{N}, 10.52 \%$. Obtained: C, 63.01\%; H, 8.50\%; N, 10.41\%.

(Z)- $N^{\prime}-\left(2,2-\right.$ diethoxyethoxy)-4-methylbenzimidamide (4d): white solid: $\mathrm{mp} 50-51{ }^{\circ} \mathrm{C}$; IR ( $\mathrm{KBr}$ pellet): 国 $\max _{\max } 822,930,1062,1303,1396,1462,1520,1622,2362,2967,2930,2973,3358,3471 \mathrm{~cm}^{-1} ;{ }^{1} \mathrm{H}$ NMR $(400$ $\left.\mathrm{MHz}, \mathrm{CDCl}_{3}\right) \delta 1.24\left(6 \mathrm{H}, \mathrm{t}, J=7.2 \mathrm{~Hz},-\mathrm{CH}_{2} \mathrm{CH}_{3}\right), 2.37\left(3 \mathrm{H}, \mathrm{s}, \operatorname{Aryl}-\mathrm{CH}_{3}\right), 3.61\left(2 \mathrm{H}, \mathrm{dq}, J=9.6\right.$ and $\left.7.2 \mathrm{~Hz},-\mathrm{CH}_{2} \mathrm{CH}_{3}\right)$, $3.76\left(2 \mathrm{H}, \mathrm{dq}, J=9.6\right.$ and $\left.7.2 \mathrm{~Hz},-\mathrm{CH}_{2} \mathrm{CH}_{3}\right), 4.11\left(2 \mathrm{H}, \mathrm{d}, J=5.6 \mathrm{~Hz},-\mathrm{CH}_{2} \mathrm{CH}-\right), 4.46\left(1 \mathrm{H}, \mathrm{t}, J=5.6, \mathrm{~Hz},-\mathrm{CH}_{2} \mathrm{CH}\right)$, $4.85(2 \mathrm{H}$, br s, NH 2$), 7.19\left(2 \mathrm{H}, \mathrm{d}, J=8.4 \mathrm{~Hz}, \mathrm{H}_{\text {Aryl }}\right), 7.52\left(2 \mathrm{H}, \mathrm{d}, J=8.4 \mathrm{~Hz}, \mathrm{H}_{\text {Aryl }}\right) ;{ }^{13} \mathrm{C} \mathrm{NMR}\left(100 \mathrm{MHz}, \mathrm{CDCl}_{3}\right) \delta$ $15.4,21.3,62.4,73.4,100.4,125.7,129.2,129.5,139.9,152.4$. Anal. calcd for $\mathrm{C}_{14} \mathrm{H}_{22} \mathrm{~N}_{2} \mathrm{O}_{3}: \mathrm{C}, 63.13 \% ; \mathrm{H}$, 8.33\%; N, 10.52\%. Obtained: C, 62.99\%; H, 8.54\%; N, 10.63\%.

(Z)-4-chloro-N'-(2,2-diethoxyethoxy)benzimidamide (4e): white solid: $\mathrm{mp}$ 43-44 $\mathrm{O}$; $\mathrm{IR}$ ( $\mathrm{KBr}$ pellet): 国 $\max _{\max } 834,927,1063,1301,1403,1497,1620,2662,2869,2935,2977,3349,3462 \mathrm{~cm}^{-1}$; ${ }^{1} \mathrm{H}$ NMR $(400 \mathrm{MHz}$, $\left.\mathrm{CDCl}_{3}\right) \delta 1.24\left(6 \mathrm{H}, \mathrm{t}, J=7.2 \mathrm{~Hz},-\mathrm{CH}_{2} \mathrm{CH}_{3}\right), 3.60\left(2 \mathrm{H}, \mathrm{dq}, J=9.2\right.$ and $\left.7.2 \mathrm{~Hz},-\mathrm{CH}_{2} \mathrm{CH}_{3}\right), 3.75(2 \mathrm{H}, \mathrm{dq}, J=9.2$ and $\left.7.2 \mathrm{~Hz},-\mathrm{CH}_{2} \mathrm{CH}_{3}\right), 4.10\left(2 \mathrm{H}, \mathrm{d}, J=5.2 \mathrm{~Hz},-\mathrm{CH}_{2} \mathrm{CH}-\right), 4.84\left(1 \mathrm{H}, \mathrm{t}, J=5.2 \mathrm{~Hz},-\mathrm{CH}_{2} \mathrm{CH}\right), 4.84\left(2 \mathrm{H}, \mathrm{br} \mathrm{s}, \mathrm{NH}_{2}\right), 7.36$ $\left(2 \mathrm{H}, \mathrm{d}, J=8.4 \mathrm{~Hz}, \mathrm{H}_{\text {Aryl }}\right), 7.57\left(2 \mathrm{H}, \mathrm{d}, J=8.4 \mathrm{~Hz}, \mathrm{H}_{\text {Aryl }}\right) ;{ }^{13} \mathrm{C}$ NMR $\left(100 \mathrm{MHz}, \mathrm{CDCl}_{3}\right) \delta 15.4,62.4,73.5,100.3$, 127.1, 128.8, 130.8, 135.9, 151.2. Anal. calcd for $\mathrm{C}_{13} \mathrm{H}_{19} \mathrm{ClN}_{2} \mathrm{O}_{3}$ : $\mathrm{C}, 54.45 \% ; \mathrm{H}, 6.68 \% ; \mathrm{N}, 9.77 \%$. Obtained: $\mathrm{C}$, $54.51 \% ; H, 6.77 \% ; \mathrm{N}, 9.59 \%$.

(Z)-4-bromo-N'-(2,2-diethoxyethoxy)benzimidamide (4f): white solid: $\mathrm{mp}$ 55-57 우 IR (KBr pellet): 国 $\max _{\max } 823,837,926,1009,1063,1303,1401,1493,1625,2361,2868,2930,2972,3347,3462 \mathrm{~cm}^{-1}$; ${ }^{1} \mathrm{H} \mathrm{NMR}$ $\left(400 \mathrm{MHz}, \mathrm{CDCl}_{3}\right) \delta 1.24\left(6 \mathrm{H}, \mathrm{t}, J=7.2 \mathrm{~Hz},-\mathrm{CH}_{2} \mathrm{CH}_{3}\right), 3.60\left(2 \mathrm{H}, \mathrm{dq}, J=8.8\right.$ and $\left.7.2 \mathrm{~Hz},-\mathrm{CH}_{2} \mathrm{CH}_{3}\right), 3.75(2 \mathrm{H}, \mathrm{dq}, J$ $=8.8$ and $\left.7.2 \mathrm{~Hz},-\mathrm{CH}_{2} \mathrm{CH}_{3}\right), 4.10\left(2 \mathrm{H}, \mathrm{d}, J=5.6 \mathrm{~Hz},-\mathrm{CH}_{2} \mathrm{CH}-\right), 4,84\left(1 \mathrm{H}, \mathrm{t}, J=5,2 \mathrm{~Hz},-\mathrm{CH}_{2} \mathrm{CH}-\right), 4.85(2 \mathrm{H}, \mathrm{br} \mathrm{s}$, $\left.\mathrm{NH}_{2}\right), 7.51\left(4 \mathrm{H}, \mathrm{m}, \mathrm{H}_{\text {Aryl }}\right) ;{ }^{13} \mathrm{C} \mathrm{NMR}\left(100 \mathrm{MHz} \mathrm{CDCl}_{3}\right) \delta 15.4,62.4,73.5,100.3,124.1,127.4,131.3,131.7$, 151.3. Anal. calcd for $\mathrm{C}_{13} \mathrm{H}_{19} \mathrm{BrN}_{2} \mathrm{O}_{3}$ : C, 47.14\%; $\mathrm{H}, 5.78 \%$; N, 8.46\%. Obtained: $\mathrm{C}, 47.22 \% ; \mathrm{H}, 5.71 \% ; \mathrm{N}, 8.37 \%$.

(Z)-N'-(2,2-diethoxyethoxy)-4-nitrobenzimidamide (4g): Colorless oil; IR (KBr pellet): 国 $]_{\max } 698,758$, 858, 907, 1050, 1344, 1517, 1600, 1636, 2882, 2932, 2976, 3368, $3481 \mathrm{~cm}^{-1},{ }^{1} \mathrm{H}$ NMR (400 MHz, CDCl $\left.{ }_{3}\right) 1.24$ $\left(6 \mathrm{H}, \mathrm{t}, J=7.2 \mathrm{~Hz},-\mathrm{CH}_{2} \mathrm{CH}_{3}\right), 3.61\left(2 \mathrm{H}, \mathrm{dq}, J=9.6\right.$ and $\left.7.2 \mathrm{~Hz},-\mathrm{CH}_{2} \mathrm{CH}_{3}\right), 3.76\left(2 \mathrm{H}, \mathrm{dq}, J=9.6\right.$ and $\left.7.2 \mathrm{~Hz},-\mathrm{CH}_{2} \mathrm{CH}_{3}\right)$, $4.15\left(2 \mathrm{H}, \mathrm{d}, J=5.2 \mathrm{~Hz},-\mathrm{CH}_{2} \mathrm{CH}-\right), 4.84\left(1 \mathrm{H}, \mathrm{t}, J=5.2 \mathrm{~Hz},-\mathrm{CH}_{2} \mathrm{CH}-\right) 4.94\left(2 \mathrm{H}, \mathrm{br} \mathrm{s}, 2 \mathrm{H}, \mathrm{NH}_{2}\right), 7.82(2 \mathrm{H}, \mathrm{d}, J=8.8$ $\left.\mathrm{Hz}, \mathrm{H}_{\text {Aryl }}\right), 8.24\left(2 \mathrm{H}, \mathrm{d}, J=8.8 \mathrm{~Hz}, \mathrm{H}_{\text {Aryl }}\right) ;{ }^{13} \mathrm{C} \mathrm{NMR}\left(100 \mathrm{MHz}, \mathrm{CDCl}_{3}\right) \delta 15.4,62.4,73.8,100.2,123.8,126.6$, 138.3, 148.6, 150.0. Anal. calcd for $\mathrm{C}_{13} \mathrm{H}_{19} \mathrm{~N}_{3} \mathrm{O}_{5}, \mathrm{C}, 52.52 \% ; \mathrm{H}, 6.44 \% ; \mathrm{N}, 14.13 \%$. Obtained: $\mathrm{C}, 52.69 \% ; \mathrm{H}$, $6.36 \% ; N, 14.21 \%$.

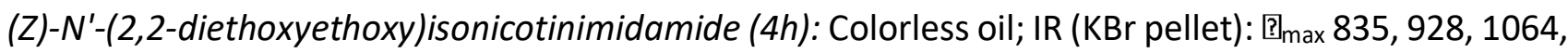
$1302,1404,1496,1624,2870,2932,2975,3349,3459 \mathrm{~cm}^{-1} ;{ }^{1} \mathrm{H}$ NMR $\left(400 \mathrm{MHz}, \mathrm{CDCl}_{3}\right) \delta 1.22(6 \mathrm{H}, \mathrm{t}, \mathrm{J}=7.2$ $\left.\mathrm{Hz},-\mathrm{CH}_{2} \mathrm{CH}_{3}\right), 3.59\left(2 \mathrm{H}, \mathrm{dq}, J=9.6\right.$ and $\left.7.2 \mathrm{~Hz}, 2 \mathrm{H},-\mathrm{CH}_{2} \mathrm{CH}_{3}\right), 3.73\left(2 \mathrm{H}, \mathrm{dq}, J=9.6\right.$ and $\left.7.2 \mathrm{~Hz},-\mathrm{CH}_{2} \mathrm{CH}_{3}\right), 4.11$ $\left(2 \mathrm{H}, \mathrm{d}, J=5.6 \mathrm{~Hz},-\mathrm{CH}_{2} \mathrm{CH}\right), 4.83\left(1 \mathrm{H}, \mathrm{t}, J=5.6 \mathrm{~Hz},-\mathrm{CH}_{2} \mathrm{CH}-\right), 4.99\left(2 \mathrm{H}, \mathrm{br} \mathrm{s}, \mathrm{NH}_{2}\right), 7.51(2 \mathrm{H}, \mathrm{dd}, J=4.8$ and 2.4

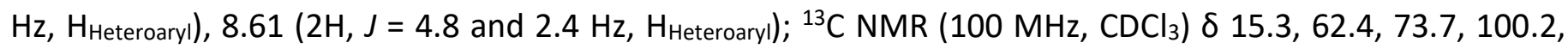


119.9, 139.8, 149.7, 150.1. Anal. calcd for $\mathrm{C}_{12} \mathrm{H}_{19} \mathrm{~N}_{3} \mathrm{O}_{3}, \mathrm{C}, 56.90 \% ; \mathrm{H}, 7.56 \% ; \mathrm{N}, 16.59 \%$. Obtained: $\mathrm{C}, 56.99 \%$; H, 7.42\%; N, 16.70\%.

\section{Fungal strains}

Candida guilliermondii ATCC 6260, Candida albicans ATCC 76615 and Candida albicans ATCC 76485 were provided by the fungal culture collection from the Laboratório de Micologia da Universidade Federal da Paraíba, Brazil.

\section{Minimum inhibitory concentration (MIC)}

The determination of minimum inhibitory concentration (MIC) was performed by broth microdilution according to CLSI (Clinical and Laboratory Standards Institute) document M27-A2 (CLSI 2017). The culture medium used was Sabouraud Dextrose Agar. The inoculum of each of the microorganisms was prepared according to the $0.5 \mathrm{McF}$ arland standard containing approximately $1-5 \times 10^{6} \mathrm{CFU} \cdot \mathrm{mL}^{-1}$ (colony forming units. $\mathrm{mL}^{-1}$ ). Experiments were conducted at approximately $1-5 \times 10^{5} \mathrm{CFU} \cdot \mathrm{mL}^{-1}$ in each well. The solutions with compounds 3a-h were prepared at the time of the tests. Dimethylsulfoxide (DMSO) was used to solubilize compounds 3a-h (the concentration of DMSO was always less than $0.5 \%$ ). Compounds $3 a-h$ was tested at concentrations ranging from 512 to $8 \mu \mathrm{g} \cdot \mathrm{mL}^{-1}$ at $1: 2$ serial dilutions. Initially, $100 \mu \mathrm{l}$ of the culture medium was added to each well of a plate. Then, a $100 \mu \mathrm{L}$ of the solution of compounds 3a-h (final concentration $512 \mu \mathrm{g} \cdot \mathrm{mL}^{-1}$ ) was added to the first line of the plate and a 1:2 serial dilution to $1 \mu \mathrm{g} \cdot \mathrm{mL}^{-1}$ concentration was performed. Finally, $10 \mu \mathrm{L}$ of the inoculum was added to each well of the plate. The plate was incubated at $35^{\circ} \mathrm{C}$ for $24-48$ hours. The minimum inhibitory concentration was considered the lowest concentration capable of inhibiting the visible growth of the microorganism. The experiment was performed in triplicate and controls with DMSO (culture medium + inoculum + DMSO) at the same concentration used to solubilize 3a-h were prepared to demonstrate that the growth of the microorganism is not influenced by DMSO.

MIC was considered the lowest concentration of the substances capable of causing visual inhibition of the growth of the strains used in the microbiologic assays, being confirmed by the addition of $20 \mu \mathrm{L}$ of $2.0 \%$ TTC (2,3,5 triphenyl tetrazolium chloride) in each cavity.

\section{Results and Discussion}

The general strategy for the synthesis of the new antifungal agents involved two reaction steps, the preparation of the amidoximes 2 from different nitriles followed by the alkylation of the obtained compounds to afford the $O$-alkylamidoximes. The synthesis started from the reaction of commercially available nitriles 1a-h and hydroxylamine hydrochloride under sonication (Barros et al. 2011; Andrade et al. 2016). In all cases, the corresponding amidoximes $2 a-h$ were obtained in short reaction times and moderate to high yields after purification by crystallization (Figure 1). The characterization data of the obtained compounds are in accordance with the literature (Li et al. 2013; Ozcan et al. 2013; Andrade et al. 2016; Tarasenko et al. 2017). 
<smiles>N#Cc1ccc(C(=O)ON)cc1</smiles>

1a-h<smiles>N/C(=N\O)c1ccccc1</smiles>

2a

$(73 \% ; 15$ min.)<smiles>N/C(=N\O)c1ccc(Cl)cc1</smiles>

$2 \mathbf{e}$

$(80 \% ; 45 \mathrm{~min}$.<smiles>Cc1ccccc1/C(N)=N/O</smiles>

2b

$(55 \% ; 45$ min.)<smiles>N/C(=N\O)c1ccc(Br)cc1</smiles>

$2 f$

$(80 \% ; 45 \mathrm{~min}$.<smiles>Cc1cccc(/C(N)=N/O)c1</smiles>

2c

$(71 \% ; 30 \mathrm{~min}$.<smiles>N/C(=N\O)c1ccc([N+](=O)[O-])cc1</smiles>

$\mathbf{2 g}$

$(91 \% ; 40 \mathrm{~min}$.<smiles>Cc1ccc(/C(N)=N/O)cc1</smiles>

2d

$(82 \% ; 20 \mathrm{~min}$.

Figure 1. Synthesis of amidoximes.

Thus, compound 2a was prepared at the shortest reaction time probably due to its high solubility in the reaction medium and lower steric hindrance. Steric factors are also important, the ortho isomer $2 \mathrm{~b}$ was obtained in lower yield and longer reaction time when compared to the meta isomers and para isomers, $2 \mathrm{c}$ and $2 \mathrm{~d}$, respectively. When halogens were present in the aromatic ring the corresponding compounds $2 \mathrm{e}$ and $2 f$ were obtained in higher yields. The best result was observed when the nitro group, a strong electronwithdrawing group was used, where compound $2 \mathrm{~g}$ was obtained in $91 \%$ yield. The method proved also to be efficient for the synthesis of heteroaromatic compounds, where $2 \mathrm{~h}$ was obtained in good yield after a half-hour.

The $O$-alkylation of amidoximes has few examples described in the literature (Coviello 1964; Veerman et al. 2016). In this way, the synthesis of alkylated amidoximes was revisited to find the best condition for this reaction. The desired compounds, 3a-h were obtained in moderate to good yields after 2 to 7 hours depending on the group present in the aromatic ring (Figure 2).<smiles>N/C(=N\O)c1ccc([GeH2])cc1</smiles><smiles>CCOC(CBr)CO[Na]</smiles><smiles>CCOC(CO/N=C(\N)c1ccccc1)OCC</smiles><smiles>CCOC(CO/N=C(\N)c1ccccc1)OCC</smiles>

3a $(79 \%, 3 \mathrm{~h})$<smiles>CCOC(CO/N=C(\N)c1ccccc1C)OCC</smiles>

3b

$(69 \%, 3 \mathrm{~h})$<smiles>CCOC(CO/N=C(\N)c1cccc(C)c1)OCC</smiles>

(76\%, $3 \mathrm{~h})$<smiles>CCOC(CO/N=C(\N)c1ccc(C)cc1)OCC</smiles>

$(67 \%, 3.5 \mathrm{~h})$<smiles>CCOC(CO/N=C(\N)c1ccc(Cl)cc1)OCC</smiles>

$3 e$<smiles>CCOC(CO/N=C(\N)c1ccc(Br)cc1)OCC</smiles><smiles>CCOC(CO/N=C(\N)c1ccc([N+](=O)[O-])cc1)OCC</smiles><smiles>CCOC(CON=C(N)c1ccncc1)OC</smiles>

$3 \mathbf{h}$

Figure 2. Synthesis of $O$-alkylated-amidoximes. 
Compounds 3a-h were then submitted to antifungal activity against three Candida species due to their prevalence in the epidemiology of fungal infections (Whaley et al. 2017) using the broth microdilution method (CLSI 2017). Thus, the Minimum Inhibitory Concentration (MIC) (Espinel-Ingroff et al. 2005) capable of visually inhibiting $100 \%$ of fungal growth for the synthesized compounds was evaluated. The results are depicted in Table 1.

Table 1. Minimum inhibitory concentrations for compounds 3a-h.

\begin{tabular}{|c|c|c|c|c|}
\hline \multirow[b]{2}{*}{ Entry } & \multirow[b]{2}{*}{ Compound } & \multicolumn{3}{|c|}{$\mathrm{MIC}, \mathrm{mM}\left(\mathrm{mg} \cdot \mathrm{mL}^{-1}\right)$} \\
\hline & & $\begin{array}{c}\text { Candida guilliermondii } \\
\text { ATCC } 6260\end{array}$ & $\begin{array}{c}\text { Candida albicans } \\
\text { ATCC } 76615\end{array}$ & $\begin{array}{c}\text { Candida albicans } \\
\text { ATCC } 76485\end{array}$ \\
\hline 1 & $3 a$ & $2.03(512)$ & $2.03(512)$ & $>2.03(>512)$ \\
\hline 2 & $3 b$ & $1.92(512)$ & $1.92(512)$ & $>1.92(>512)$ \\
\hline 3 & $3 c$ & $>1.92(>512)$ & $1.92(512)$ & $>1.92(>512)$ \\
\hline 4 & $3 d$ & $1.92(512)$ & $>1.92(>512)$ & $>1.92(>512)$ \\
\hline 5 & $3 e$ & $1.79(512)$ & $1.79(512)$ & $1.79(512)$ \\
\hline 6 & $3 f$ & $1.55(512)$ & $1.55(512)$ & $>1.55(>512)$ \\
\hline 7 & $3 g$ & $0.86(256)$ & $0.86(256)$ & $1.72(512)$ \\
\hline 8 & $3 h$ & $>2.02(>512)$ & $2.02(512)$ & $>2.02(>512)$ \\
\hline $9^{a}$ & - & $0.11(32)$ & $0.11(32)$ & $0.83(256)$ \\
\hline
\end{tabular}

a Fluconazole was used as a positive control.

In general, all compounds shown antifungal activity against at least one strain of Candida. As described by Morales et al. (2008) a compound with poor antimicrobial activity shows MIC values above of $1500 \mu \mathrm{g} \cdot \mathrm{mL}^{-1}$, moderate activity in the range of 500-1500 $\mathrm{gg} \cdot \mathrm{mL}^{-1}$, and good activity with MIC values in the range of $50-500 \mu \mathrm{g} \cdot \mathrm{mL}^{-1}$. It is also interesting to note that the position of the substituents attached to the aromatic ring did not imply significant changes in MIC values. For example, compounds $3 \mathrm{~b}-\mathrm{d}$ which contain a methyl group at the ortho, meta, or para positions, respectively, exhibited similar MIC values (Table 1, entries 2-4). Aromatic rings containing a halogen as substituent $3 e$ and $3 f$ exhibited better activities against all strains tested (Table 1, entries 5 and 7). These findings are in accordance with the commercially available antifungal agents chlorotrimazole and econazole. Structure-activity studies have revealed that the presence of a halogen is critical for drug activity for both compounds (Kasper et al. 2015).

The best result was observed for compound $3 g$, which contains the strongly electron-withdrawing group nitro group (Table 1, entry 7). This is an interesting result, while a number of antibiotics bearing nitro groups were reported from bacteria, such as chloramphenicol and pyrrolnitrin (Al-Zereini et al. 2007) but not for antifungal agents. Finally, compound $3 \mathrm{~h}$ exhibits an antifungal activity similar to $3 \mathrm{a}$, which has no substituents at the aromatic ring (Table 1, entries 1 and 8).

One of the most important steps in the process in the development of new drugs is the prospecting of new prototypes that can be optimized based on planned molecular modifications for viable therapeutic options (Pinzi and Rastelli 2019). Further studies in the mode of action of the synthesized compounds are underway in our laboratories.

\section{Conclusions}

New $O$-alkylamidoximes were obtained in moderate to good yields (68-81\%) without any observable decomposition using a simple, reproducible, and non-expensive method. All $O$-alkylamidoximes showed in vitro antifungal activity against at least one of the tested strains of Candida. These findings are the first step in the development of new drugs, providing important information for future research to obtain new classes of antifungal agents.

Authors' Contributions: BARBOSA, M.V.S.: acquisition of data, analysis and interpretation of data; NASCIMENTO, A.K.P.: acquisition of data, analysis and interpretation of data; CAIANA, R.R.A.: acquisition of data, analysis and interpretation of data; SANTOS, C.S.: acquisition of data, analysis and interpretation of data; OLIVEIRA, W.A.: conception and design, acquisition of data, and drafting the article; MENEZES, P.H.: 
conception and design, acquisition of data, and drafting the article; FREITAS, J.C.R.: conception and design, acquisition of data, and drafting the article. All authors have read and approved the final version of the manuscript.

Conflicts of Interest: The authors declare no conflicts of interest.

Ethics Approval: Not applicable.

Acknowledgments: The authors would like to thank the funding for the realization of this study provided by the Brazilian agencies FACEPE (Fundação de Amparo a Ciência e Tecnologia de Pernambuco - Brasil), through the PRONEM (Programa de Apoio a Núcleos Emergentes), Finance Code APQ-0476-1.06/14, and CNPq (Conselho Nacional de Desenvolvimento Científico e Tecnológico - Brasil), Finance Code 434012/2018-1. The authors are also grateful to the Analytical Center of the Department of Fundamental Chemistry at the Federal University of Pernambuco for the analysis of the synthesized compounds.

\section{References}

AL-ZEREINI, W., et al. New aromatic nitro compounds from Salegentibacter sp. T436, an Arctic Sea ice bacterium: taxonomy, fermentation, isolation and biological activities. The journal of Antibiotico. 2007, 60(5), 301-308. https://doi.org/10.1038/ja.2007.38

ANDRADE, D., FREITAS FILHO, J.R. and FREITAS, J.C.R. Aplicação de amidoximas como catalisadores da reação de alilação por ali ltrifluoroborato de potássio em meio bifásico. Quimica Nova. 2016, 39(10), 1225-1235. https://doi.org/10.21577/0100-4042.20160158

ARMAREGO, W.L.F. Purification of Laboratory Chemicals. 8a ed. Amsterdam: Butterworth Heinemann, 2017.

BARROS, C.J.P., et al. Synthesis of amidoximes using an efficient and rapid ultrasound method. Journal of the Chilean Chemical Society. 2011, 56(2), 721-722. https://doi.org/10.4067/S0717-97072011000200022

BERMAN, J. and KRYSAN, D.J. Drug resistance and tolerance in fungi. Nature Reviews Microbiology. 2020, 15, 1-13.

https://doi.org/10.1038/s41579-019-0322-2

BOYKIN, D.W., et al. Anti-pneumocystis activity of bis-amidoximes and bis-o-alkylamidoximes prodrugs. Bioorganic \& Medicinal Chemistry Letters. 1996, 6(24), 3017-3020. https://doi.org/10.1016/S0960-894X(96)00557-4

CAPOTE, A.M., et al. Micosis superficiales: casuística del Departamento de Micología del Instituto Nacional de Higiene "Rafael Rangel", Caracas, Venezuela (2001-2014). Investigación Clínica. 2016, 57(1), 47-48.

CAVALEIRO, C., et al. Antifungal activity of Juniperus essential oils against dermatophyte, Aspergillus and Candida strains. Journal of Applied Microbiology. 2006, 100(6), 1333-1338. https://doi.org/10.1111/j.1365-2672.2006.02862.x

Clinical and Laboratory Standards Institute (CLSI). Reference method for broth dilution antifungal susceptibility testing of yeasts. 4th ed. CLSI standard M27 Wayne, PA: Clinical and Laboratory Standards Institute, 2017.

COSTA-DE-OLIVEIRA, S. and RODRIGUES, A.G. Candida albicans antifungal resistance and tolerance in bloodstream infections: the triad yeasthost-antifungal. Microorganisms. 2020, 8(2), 154-173. https://doi.org/10.3390/microorganisms8020154

COSTELLO, E.K., et al. Bacterial community variation in human body habitats across space and time. Science. 2009, 326(5960), $1694-1697$. https://doi.org/10.1126/science.1177486

COVIELLO, D.A. Preparation of O-alkylamidoximes from $\alpha$-alkyloximinocarboxamides by the hofmann hypobromite reaction. Journal of Pharmaceutical Sciences. 1964, 53(8), 971-972. https://doi.org/10.1002/ips.2600530833

DE KRAKER, M.E.A., STEWARDSON, A.J. and HARBARTH, S. Will 10 million people die a year due to antimicrobial resistance by 2050 ? PLOS Medicine. 2016, 13(11), 1-6. https://doi.org/10.1371/journal.pmed.1002184

EADES, C.P. and ARMSTRONG-JAMES, D.P.H. Invasive fungal infections in the immunocompromised host: Mechanistic insights in an era of changing immunotherapeutics. Medical Mycology. 2019, 57(3). 307-317. https://doi.org/10.1093/mmy/myy136

ESPINEL-INGROFF, A., et al. Quality control and reference guidelines for clsi broth microdilution susceptibility method (M38-A Document) for amphotericin b, itraconazole, posaconazole, and voriconazole. Journal of Clinical Microbiology. 2005, 43(10), 5243-5246.

https://doi.org/10.1128/JCM.43.10.5243-5246.2005

IRFAN, M., et al. Effect of quinoline based 1,2,3-triazole and its structural analogues on growth and virulence attributes of Candida albicans. PLOS ONE. 2017, 12(4), 1-23. https://doi.org/10.1371/journal.pone.0175710

KAPITAN, M., et al. Fungi as part of the microbiota and interactions with intestinal bacteria. Current Topics in Microbiology and Immunology. 2019, 422, 265-301. https://doi.org/10.1007/82 2018117

KASPER, L., et al. Antifungal activity of clotrimazole against Candida albicans depends on carbon sources, growth phase and morphology. Journal of Medical Microbiology. 2015, 64(7), 714-723. https://doi.org/10.1099/jmm.0.000082

KUNG, H.C., et al. guidelines for the use of antifungal agents in patients with invasive fungal diseases in Taiwan. Journal of Microbiology, Immunology and Infection. 2016, 51(1), 1-17. https://doi.org/10.1016/j.jmii.2017.07.006

LEE, H. and LEE, D.G. Novel approaches for efficient antifungal drug action. Journal of Microbiology and Biotechnology. 2018, 28(11), 17711781. https://doi.org/10.4014/jmb.1807.07002 
LI, Y., et al. Synthesis, insecticidal activity, and structure-activity relationship (SAR) of anthranilic diamides analogs containing oxadiazole rings. Organic \& Biomolecular Chemistry. 2013, 11(24), 3979-3988. https://doi.org/10.1039/С30B40345A

MCCARTHY, M.W., et al. Novel Agents and drug targets to meet the challenges of resistant fungi. The Journal of Infectious Diseases. 2017, 216(3), 474-483. https://doi.org/10.1093/infdis/jix130

MORALES, G., et al. Antimicrobial activity of three baccharis species used in the traditional medicine of northern Chile. Molecules. 2008, 13(4), 790-794. https://doi.org/10.3390/molecules13040790

OZCAN, S., et al. Oxadiazole-isopropylamides as potent and noncovalent proteasome inhibitors. Journal of Medicinal Chemistry. 2013, 56(10), 3783-3805. https://doi.org/10.1021/jm400221d

PFALLER, M.A. Antifungal drug resistance: mechanisms, epidemiology, and consequences for treatment. The American Journal of Medicine. 2012, 125(1), 3-13. https://doi.org/10.1016/i.amjmed.2011.11.001

PINZI, L. and RASTELLI, G. Molecular Docking: Shifting Paradigms in Drug Discovery. International Journal of Molecular Sciences. 2019, 20(18), 4331-4353. https://doi.org/10.3390/ijms20184331

PRASAD, R. and KAPOOR, K. Multidrug resistance in yeast candida. International Review of Cytology. 2004, 242, 215-248.

https://doi.org/10.1016/S0074-7696(04)42005-1

REHSE, K. and BREHME, F. Amidoximes and their prodrugs. Archiv der Pharmazie - Chemistry in Life Sciences. 1998, 331(12), 375-379. https://doi.org/10.1002/(SICI)1521-4184(199812)331:12<375:AID-ARDP375>3.0.CO;2-F

SILVA, S., et al. Candida glabrata, Candida parapsilosis and Candida tropicalis: biology, epidemiology, pathogenicity and antifungal resistance. FEMS Microbiology Reviews. 2012, 36(2), 288-305. https://doi.org/10.1111/j.1574-6976.2011.00278.x

SPITZER, M., ROBBINS, N. and WRIGHT, G.D. Combinatorial strategies for combating invasive fungal infections. Virulece. 2017, 8(2), 169-185. https://doi.org/10.1080/21505594.2016.1196300

STILL, W.C., KAHN, M. and MITRA, A. Rapid chromatographic technique for preparative separations with moderate resolution. Journal of Organic Chemistry. 1978, 43(14), 2923-2925. https://doi.org/10.1021/j000408a041

SZABADOS, E., et al. BGP-15, a nicotinic amidoxime derivate protecting heart from ischemia reperfusion injury through modulation of poly (ADP-ribose) polymerase. Biochemical Pharmacology. 2000, 59(8), 937-945. https://doi.org/10.1016/s0006-2952(99)00418-9

TARASENKO, M., et al. Room-temperature synthesis of pharmaceutically important carboxylic acids bearing the 1,2,4-oxadiazole moiety. Tetrahedron Letters. 2017, 58(13), 3672-3677. https://doi.org/10.1016/j.tetlet.2017.08.020

VEERMAN, J.J.N., et al. Strategic and tactical approaches to the synthesis of 5,6-dihydro-[1,2,4]oxadiazines. Heterocycles. 2016, 92(12), 21662200. https://doi.org/10.3987/COM-16-13570

WANG, R.J., MILLER, R.F. and HUANG, L. Approach to fungal infections in human immunodeficiency virus-infected individuals: pneumocystis and beyond. Clinics in Chest Medicine. 2017, 38(3), 465-477. https://doi.org/10.1016/i.ccm.2017.04.008

WHALEY, S.G., et al. Azole antifungal resistance in Candida albicans and emerging non-albicans Candida species. Fronters Microbiology. 2017, 7, 2173-2184. https://doi.org/10.3389/fmicb.2016.02173

WIEDERHOLD, N. P. Antifungal resistance: current trends and future strategies to combat. Infection and Drug Resistance. 2017, 29(10), 249259. https://doi.org/10.2147/IDR.S124918

ZHAN-TAO, Z., et al. Syntheses and antibacterial activities of novel erythromycin O-Alkylamidoximes. Chemical Research in Chinese Universities. 2005, 21, 540-544.

Received: 25 April 2020 | Accepted: 18 September 2020 | Published: 20 August 2021

This is an Open Access article distributed under the terms of the Creative Commons Attribution License, which permits unrestricted use, distribution, and reproduction in any medium, provided the original work is properly cited. 